\title{
Recent epidemiology of neonatal sepsis in Japan: did the strategies to control and prevent MRSA transmission lead to a reduction in the incidence of late-onset sepsis?
}

\author{
This article was published in the following Dove Press journal: \\ Research and Reports in Neonatology \\ 21 October 2014 \\ Number of times this article has been viewed
}

\section{Ichiro Morioka \\ Sota Iwatani \\ Tsubasa Koda \\ Miwako Nagasaka \\ Keiji Yamana \\ Daisuke Kurokawa \\ Kaori Fujita \\ Kosuke Nishida \\ Kazumoto lijima}

Department of Pediatrics, Kobe University Graduate School of

Medicine, Kobe, Japan
Correspondence: Ichiro Morioka Department of Pediatrics, Kobe University Graduate School of Medicine, 7-5-I Kusunoki-cho, Chuo-ku, Kobe 650-0017, Japan

Tel +8I 783826090

Fax +8I 783826099

Email ichim@med.kobe-u.ac.jp

\begin{abstract}
Neonatal sepsis is a significant cause of morbidity and mortality in newborns, particularly in the preterm or low birth weight infant. In this epidemiologic review regarding neonatal sepsis, the recent epidemiology and causative pathogens in Japan are described and compared with data from international organizations. Recent Japanese multicenter studies report the incidence of neonatal sepsis at $0.74 \%$. Although the incidence of early-onset sepsis has been low $(0.13 \%)$, approximately $5 \%$ of very or extremely low birth weight infants develop late-onset sepsis with a high mortality rate. In Japan for such cases, the most predominant causative pathogen is methicillin-resistant Staphylococcus aureus; internationally, the most commonly reported pathogen is coagulase-negative staphylococci. It is our contention that the incorporation of the strategies to control and to prevent health care-associated MRSA transmission has contributed to the reduction in the incidence of late-onset sepsis.
\end{abstract}

Keywords: coagulase-negative staphylococci, incidence, methicillin-resistant Staphylococcus aureus, mortality, neonatal intensive care, nosocomial infections

\section{Introduction}

Neonatal sepsis is a significant cause of morbidity and mortality in newborns, particularly in preterm or low birth weight (LBW) infants. According to the World Health Organization estimates, there are four million neonatal deaths worldwide every year. Over $35 \%$ of these are in underdeveloped countries where mortality due to sepsis may be as high as $85 \%$; the mortality has consistently been about $20 \%$ in developed countries. ${ }^{1-5}$

Neonatal sepsis with a high mortality rate mainly occurs in preterm newborns in developed countries. ${ }^{1,5-7}$ Preterm newborns are at high risk for developing infection due to their immunologic immaturity. ${ }^{8}$ Additionally, some studies for preterm infants have demonstrated a strong association between sepsis and periventricular leukomalacia, or cerebral white matter damage. ${ }^{9-11}$ Compared with uninfected extremely low birth weight (ELBW) infants weighing $<1,000 \mathrm{~g}$ at birth, those with sepsis had significantly more adverse neurodevelopmental outcomes at follow-up, including: cerebral palsy (odds ratio [OR], 1.4); low Bayley Scales of Infant Development II scores on the mental development index (OR, 1.3) and the Psychomotor Developmental Index (OR, 1.5); and vision impairment $(\mathrm{OR}, 1.7) .{ }^{9}$ Neonatal sepsis remains a major disease worldwide in need of improved outcomes. ${ }^{12}$

The purpose of this review is to present the recent epidemiology and pathogens of neonatal sepsis in Japan. Because methicillin-resistant Staphylococcus aureus (MRSA) 
is a key pathogen in the incidence of neonatal sepsis, we also describe the strategies to mitigate the risk of MRSA transmission in Japanese neonatal intensive care units (NICUs).

\section{Setting}

Japanese NICUs usually consist of the units of levels II and III intensive cares. Level II units include the beds for patients who needed transitional care, such as LBW infants, newborn patients with mild diseases, or patients after their treatment of acute states of the disease in level III units. Level III units include the beds for patients who required more intensive care than those in level II units, such as very low birth weight (VLBW) infants weighing $<1,500 \mathrm{~g}$ at birth, ELBW infants, and newborns who need some treatments for underlying diseases. The patient:nurse ratio is 1:6 for level II care and 1:3 for level III care.

In this article, NICU includes both units of levels III and II intensive cares. Most NICUs accept not only the preterm and sick newborns that are born in their own hospital, but they also accept those who are transferred from general hospitals or obstetrical clinics in the surrounding area. Some of them accept newborn patients who need surgery, inhaled nitric oxide, and extracorporeal membrane oxygenation.

The central venous line, nasogastric tube placement, endotracheal intubation, pulse oximeter, electrocardiography, phototherapy device, and incubator are used as routine treatments and managements in every NICU. Policies of use of a central venous line and antibiotics are shown as follows: ${ }^{5}$

\section{Use of a central venous line}

- For maintaining a central venous line, peripherally inserted central catheters are used. When a central venous line using peripherally inserted central catheters cannot be successfully placed because of ELBW infants or newborns with severe asphyxia, umbilical vein catheters are used instead.

- Preterm newborns, such as those with a birth weight $<1,800$ g or a gestational age of $<34$ weeks, those with intestinal disorders, those who need total parenteral nutrition, and those who have drugs that cause angiitis use a central venous line.

- When the status of newborns is improved, central venous lines are immediately removed.

\section{Use of antibiotics}

- For newborns with suspected infection or sepsis, antibiotics are used for treatment. Prophylactic antibiotics are sometimes used for VLBW infants and newborns with respirators or central venous lines.
- Penicillin or oxacephem is used as the first choice for early-onset infection. Aminoglycoside is added when required for treatment.

- For late-onset infection, the specific antibiotic choice is driven by center-specific guidelines based on the major cause of nosocomial infection and organism susceptibility patterns in that specific center.

- De-escalation for use of specific antibiotics (definitive therapy) is performed when a targeted organism is found by culture.

\section{Definition and classification of neonatal sepsis}

Neonatal sepsis is generally defined as a clinical syndrome characterized by signs and symptoms of infection in addition to growth of an organism from the blood..$^{67,13}$ Most of the causative agents are bacteria, viruses, and fungi that, on the earliest presentation, may be associated with any gradation of symptoms, from only subtle feeding disturbances to frank septic shock. ${ }^{14}$ In the Japanese NICUs, the definition of neonatal sepsis is one or more of the following clinical symptoms: fever; hypothermia; temperature instability; apnea; bradycardia; respiratory distress; increased oxygen requirements; feeding intolerance; lethargy or hypotonia; and a blood culture positive for a single organism. ${ }^{5}$ When bacteria that occur naturally on the skin are detected by blood culture, the same bacteria should be detected from blood at least twice.

Neonatal sepsis can be classified into two major categories - early-onset sepsis (EOS) and late-onset sepsis (LOS), depending on the timing of onset of signs/symptoms. The definitions of EOS and LOS vary. In Japan, we usually define EOS as the onset of signs/symptoms and a positive culture result within 72 hours, and LOS as those after 72 hours of age. ${ }^{5,15}$ These classifications of EOS and LOS reflect the differing etiologies and pathophysiology of pathogens. ${ }^{6}$ While EOS is usually associated with the transmission of organisms from mother to newborn, LOS is usually due to organisms acquired after birth via the hands of health care personnel, which is called a nosocomial (health care-associated) transmission. ${ }^{16-19}$

\section{Recent epidemiology Incidence}

Because a nationwide surveillance system for neonatal sepsis is currently underdeveloped in Japan, there are few recent published reports on the epidemiology and pathogens of neonatal sepsis. We investigated the morbidity and mortality of culture-proven neonatal sepsis and pathogen 
characteristics in five Japanese NICUs with 6,894 admitted newborns from 2006-2008. ${ }^{5}$ The total incidence of neonatal sepsis was $0.74 \%$ (EOS, $0.13 \%$; LOS, $0.61 \%$ ). The incidence of EOS was extremely low in Japan, because there was no EOS with group B Streptococcus (GBS) and an extremely low incidence of EOS with Escherichia coli during the study period. GBS and E. coli are still the most common pathogens associated with EOS worldwide. GBS currently comprises $40 \%-50 \%$ of the total pathogens of EOS in England and the US because of missed opportunities for the prevention of GBS vertical transmission. ${ }^{20,21}$

A recent nationwide surveillance study in Japan has also shown that the estimated incidence of early-onset GBS disease was $0.08 / 1,000$ live births. ${ }^{22}$ The incidence of earlyonset GBS disease is apparently low in Japan, because the management of pregnant women with GBS based on the recommendations from the US Centers for Disease Control and Prevention (CDC) may have become standard and mostly followed in Japan. ${ }^{23}$ Furthermore, no difference between each NICU was observed for the incidence of EOS in a study of Japanese NICUs. ${ }^{5}$

However, LOS occurred in 21 (5.6\%) of 378 ELBW infants with a mortality rate of $29 \%$ in the study of five Japanese NICUs (Table 1). ${ }^{5}$ A large multicenter survey report, including 37 Japanese NICUs of level III intensive cares and 2,145 VLBW infants, revealed that the incidence of sepsis was $8 \%$ in $2003 .{ }^{24}$ The Neonatal Research Network of Japan also reported that $492(5.0 \%)$ of 9,812 VLBW infants, who were born from 2006-2008, developed LOS (Table 1). ${ }^{25}$ A current issue is how to control and prevent LOS in VLBW infants in Japan. However, the incidence of LOS in VLBW infants was considerably lower than that in the US and Canada (Table 1). ${ }^{25,26}$

\section{Pathogens causing LOS}

In the study of five Japanese NICUs in 2006-2008, the most common causative pathogen of LOS was $S$. aureus (mainly MRSA), followed by methicillin-resistant coagulase-negative staphylococci (MRCoNS), Pseudomonas aeruginosa, and

Table I Recent incidence of late-onset neonatal sepsis in preterm infants in Japan, the US, and Canada

\begin{tabular}{|c|c|c|c|c|}
\hline & \multicolumn{2}{|l|}{ Japan $^{5,25}$} & \multirow{2}{*}{$\begin{array}{l}\frac{U^{26}}{\text { VLBWI }} \\
n=20,472\end{array}$} & \multirow{2}{*}{$\begin{array}{l}\text { Canada }^{25} \\
\text { VLBWI } \\
n=5,34 I\end{array}$} \\
\hline & $\begin{array}{l}\text { ELBWI } \\
n=378\end{array}$ & $\begin{array}{l}\text { VLBWI } \\
n=9,8 I 2\end{array}$ & & \\
\hline Years & $2006-2008$ & $2006-2008$ & $2002-2008$ & $2006-2008$ \\
\hline $\begin{array}{l}\text { Late-onset } \\
\text { sepsis (\%) }\end{array}$ & 5.6 & 5.0 & 24.4 & 16.6 \\
\hline
\end{tabular}

Abbreviations: ELBWI, extremely low birth weight infants; VLBWI, very low birth weight infants.
E. coli (Table 2). ${ }^{5}$ In the deceased cases, the most common causative pathogen was MRSA, followed by P. aeruginosa. ${ }^{5}$ The most common pathogen differed between Japan and the other countries mentioned in the study, which was MRSA in Japan and CoNS in other countries, such as the US, along with countries in Europe and Asia. ${ }^{1,20,26,27}$ MRSA is sometimes transmitted from infants to infants when there are many infants with MRSA colonization in the NICU, even though some precautions are taken for MRSA-colonized infants. We need to reduce the number of infants that are carrying MRSA in each NICU.

\section{History of the spread of MRSA in Japan}

The pathogens in neonatal sepsis in Japan are unique compared with those in other countries. MRSA was a major pathogen in neonatal sepsis, mainly LOS in ELBW or VLBW infants. The reason for the difference could be that the rate of methicillin resistance among $S$. aureus strains in many Japanese general hospitals was $>60 \%-70 \%$, and $>50 \%$ of Japanese NICUs have taken care of infants with MRSA colonization. ${ }^{16}$

The mortality rates for ELBW infants have been markedly improved in Japan since the year 2000. ${ }^{28}$ At the same time, in the early 2000s, MRSA widely spread into most Japanese NICUs. ${ }^{16,18}$ This was a serious problem because $87 \%$ of major Japanese NICUs had patients with MRSA infection in 2000, ${ }^{16,18}$ and the mortality rate due to health care-associated MRSA infection was $15 \%$ in VLBW infants. ${ }^{18}$ In recent years, the incidence of LOS in VLBW infants is considerably lower than that in other developed countries. We speculated that the reasons for the reduction in incidence are because of the widespread

Table 2 Pathogens of late-onset neonatal sepsis in the study of five Japanese NICUs in 2006-2008

\begin{tabular}{ll}
\hline & Numbers (\%) \\
& $\mathbf{n = 4 2}$ \\
\hline Staphylococcus aureus & $\mathrm{II}(26 \%)$ \\
$\quad$ MRSA & $8(19 \%)$ \\
MSSA & $3(7 \%)$ \\
Methicillin-resistant coagulase-negative staphylococci & $5(12 \%)$ \\
Pseudomonas aeruginosa & $5(12 \%)$ \\
Escherichia coli & $5(12 \%)$ \\
Group B Streptococcus & $3(7 \%)$ \\
Group A Streptococcus & $3(7 \%)$ \\
Enterobacter cloacae & $2(5 \%)$ \\
Klebsiella pneumoniae & $2(5 \%)$ \\
Others & $6(14 \%)$ \\
\hline
\end{tabular}

Note: Copyright (C) 2012. S Karger AG, Basel, Switzerland. Reproduced from Morioka I, Morikawa S, Miwa A, et al. Culture-proven neonatal sepsis in Japanese neonatal care units in 2006-2008. Neonatology. 2012;102(I):75-80. ${ }^{5}$

Abbreviations: NICU, neonatal intensive care units; MRSA, methicillin-resistant S. aureus; MSSA, methicillin-sensitive S. aureus. 
use of the guidelines for hand hygiene and the management of multidrug-resistant organisms, including MRSA, published from the CDC for the years 2002 and 2006, respectively. ${ }^{29,30}$

The CDC recommendations for management of MRSA are as follows: improvements in the compliance with hand hygiene (it is important to have correct hand hygiene technique with accurate timing); contact precautions (use of gloves with disposable gowns or aprons for all actions that may involve contact with the patient with MRSA, or isolation); active surveillance cultures; education for medical staffs, families, and community; enhanced environmental cleaning; and communications about patients with MRSA within and between health care facilities. ${ }^{30}$

\section{Changes in prevalence of MRSA- colonized infants and measures to control and prevent health care- associated MRSA transmission in Japanese NICUs}

A nationwide survey was conducted in Japanese NICUs in 2000 and 2011 by the Japan Society of Premature and Newborn Medicine. ${ }^{16}$ The surveyed NICUs in 2000 and 2011 were not the same. However, we can observe trends in the proportion of MRSA-colonized patients and changes in measures to control and to prevent health care-associated MRSA transmission. The percentage of NICUs reporting as free of MRSA-colonized patients increased from 14\% of 65 responding NICUs in 2000 to $53 \%$ of 150 responding NICUs in 2011 (Table 3). ${ }^{16}$ The proportion of NICUs where the clinical staff used gloves when caring for patients, and gathering (cohorting) of MRSA colonized infants was practiced increased from the year 2000. The use of mupirocin to eradicate MRSA for colonized patients, the use of mupirocin to eradicate MRSA for the clinical staff carrying MRSA, use

Table 3 Changes in prevalence of MRSA-colonized infants and measures to control and prevent health care-associated MRSA transmission in Japanese NICUs

\begin{tabular}{lll}
\hline Year & 2000 & 2011 \\
& 65 NICUs & 150 NICUs \\
\hline
\end{tabular}

Prevalence of MRSA-co lonized infants

Infants without MRSA $\quad 14 \% \quad 53 \%$

colonization in NICU

Note: Reproduced with permission from John Wiley and Sons. Prevalence of MRSA colonization in Japanese neonatal care unit patients in 201 I. Morioka I, Takahashi N, Kitajima H; Committee for Infection Prevention and Vaccine Promotion of the Japan Society for Premature and Newborn Medicine. Pediatr Int. 2014;56(2):2II-214.16 Copyright (c) 2013 Morioka I, Takahashi N, Kitajima H. Pediatrics International @ 2013 Japan Pediatric Society.

Abbreviations: MRSA, methicillin-resistant Staphylococcus aureus; NICU, neonatal intensive care unit.
Table 4 Measures to control and prevent health care-associated MRSA transmission

\begin{tabular}{lll}
\hline Year & $\mathbf{2 0 0 0}$ & $\mathbf{2 0 I}$ I \\
& $\mathbf{9 1}$ NICUs & $\mathbf{1 6 8 \text { NICUs }}$ \\
\hline Measures & & \\
$\quad$ Glove use & $41 \%$ & $90 \%$ \\
$\quad$ Cohorting/isolation for MRSA+ infants & $58 \%$ & $73 \%$ \\
$\quad$ Mupirocin for MRSA+ infants & $56 \%$ & $36 \%$ \\
$\quad$ Povidone iodine for umbilical cord & $35 \%$ & $0 \%$ \\
Disinfectants for hand hygiene & & \\
Alcohol & $0 \%$ & $89 \%$ \\
$\quad$ Soap & $12 \%$ & $73 \%$ \\
$\quad$ Povidone iodine & $71 \%$ & $21 \%$ \\
\hline
\end{tabular}

Note: Reproduced with permission from John Wiley and Sons. Prevalence of MRSA colonization in Japanese neonatal care unit patients in 20II. Morioka I, Takahashi N, Kitajima H; Committee for Infection Prevention and Vaccine Promotion of the Japan Society for Premature and Newborn Medicine. Pediatr Int. 2014;56(2):2II-2/4.16 Copyright () 2013 Morioka I, Takahashi N, Kitajima H. Pediatrics International @ 2013 Japan Pediatric Society.

Abbreviations: MRSA, methicillin-resistant Staphylococcus aureus; NICU, neonatal intensive care unit.

of a bath with disinfectant for MRSA-colonized patients, and the disinfection of the umbilical cord by povidone iodine decreased in this decade. (Bathing with disinfectant for MRSA-colonized patients and disinfection of the umbilical cord by povidone iodine were experience-based measures). Disinfectant agents for hand hygiene for the clinical staff changed from povidone iodine to an alcohol-based hand rub and soap in the last decade. In $>85 \%$ of NICUs in 2011 , working clinical staff used an alcohol-based hand rub and wore gloves to control and prevent health care-associated MRSA transmission (Table 4). ${ }^{16}$

The proportion of NICUs that have MRSA-colonized infants has decreased, and the control and preventive measures for MRSA colonization dramatically changed in the last decade. We propose that the recommendations on hand hygiene using alcohol and the management of multidrug-resistant organisms published by the CDC have contributed to this improvement. ${ }^{29,30}$ As a result, the incidence of neonatal sepsis, including LOS with MRSA in Japan, may have decreased in recent years.

\section{Conclusion}

Approximately 5\% of VLBW or ELBW infants developed LOS, and the most common causative pathogen of LOS was MRSA in 2006-2008. When LOS occurred in ELBW infants, the mortality rate was still high. Since the proportion of NICUs with MRSA-colonized infants has decreased because of the changes in measures to control and to prevent health care-associated MRSA transmission, further reduction of the incidence of neonatal sepsis with MRSA may be achievable in Japan in the near future. 


\section{Acknowledgment}

This work was supported by Grant-in-Aid for Scientific Research (C) of JSPS KAKENHI grant number 26461632.

\section{Disclosure}

The authors report no conflicts of interest in this work.

\section{References}

1. Hornik CP, Fort P, Clark RH, et al. Early and late onset sepsis in verylow-birth-weight infants from a large group of neonatal intensive care units. Early Hum Dev. 2012;88 Suppl 2:S69-S74.

2. Lawn JE, Wilczynska-Ketende K, Cousens SN. Estimating the causes of 4 million neonatal deaths in the year 2000. Int J Epidemiol. 2006;35(3): 706-718.

3. Sankar MJ, Agarwal R, Deorari AK, Paul VK. Sepsis in the newborn. Indian J Pediatr. 2008;75(3):261-266.

4. Satar M, Ozlü F. Neonatal sepsis: a continuing disease burden. Turk $J$ Pediatr. 2012;54(5):449-457.

5. Morioka I, Morikawa S, Miwa A, et al. Culture-proven neonatal sepsis in Japanese neonatal care units in 2006-2008. Neonatology. 2012;102(1): 75-80.

6. Shane AL, Stoll BJ. Recent developments and current issues in the epidemiology, diagnosis, and management of bacterial and fungal neonatal sepsis. Am J Perinatol. 2013;30(2):131-141.

7. Gardner SL. Sepsis in the neonate. Crit Care Nurs Clin North Am. 2009;21(1):121-141, vii.

8. Wynn JL, Levy O. Role of innate host defenses in susceptibility to early-onset neonatal sepsis. Clin Perinatol. 2010;37(2):307-337.

9. Stoll BJ, Hansen NI, Adams-Chapman I, et al; National Institute of Child Health and Human Development Neonatal Research Network. Neurodevelopmental and growth impairment among extremely low-birthweight infants with neonatal infection. JAMA. 2004;292(19):2357-2365.

10. Shah DK, Doyle LW, Anderson PJ, et al. Adverse neurodevelopment in preterm infants with postnatal sepsis or necrotizing enterocolitis is mediated by white matter abnormalities on magnetic resonance imaging at term. J Pediatr. 2008;153(2):170-175, 175. e1.

11. Volpe JJ. Postnatal sepsis, necrotizing entercolitis, and the critical role of systemic inflammation in white matter injury in premature infants. J Pediatr. 2008;153(2):160-163.

12. Bizzarro MJ, Raskind C, Baltimore RS, Gallagher PG. Seventy-five years of neonatal sepsis at Yale: 1928-2003. Pediatrics. 2005;116(3):595-602.

13. Vergnano S, Sharland M, Kazembe P, Mwansambo C, Heath PT. Neonatal sepsis: an international perspective. Arch Dis Child Fetal Neonatal Ed. 2005;90(3):F220-F224.

14. Baltimore RS. Neonatal sepsis: epidemiology and management. Paediatr Drugs. 2003;5(11):723-740.

15. Usukura Y, Igarashi T. Examination of severe, hospital acquired infections affecting extremely low birthweight (ELBW) infants. Pediatr Int. 2003;45(2):230-232.

16. Morioka I, Takahashi N, Kitajima H; Committee for Infection Prevention and Vaccine Promotion of the Japan Society for Premature and Newborn Medicine. Prevalence of MRSA colonization in Japanese neonatal care unit patients in 2011. Pediatr Int. 2014;56(2):211-214.
17. Mulligan ME, Murray-Leisure KA, Ribner BS, et al. Methicillin-resistant Staphylococcus aureus: a consensus review of the microbiology, pathogenesis, and epidemiology with implications for prevention and management. Am J Med. 1993;94(3):313-328.

18. Kitajima H. Prevention of methicillin-resistant Staphylococcus aureus infections in neonates. Pediatr Int. 2003;45(2):238-245.

19. Strausbaugh LJ, Siegel JD, Weinstein RA. Preventing transmission of multidrug-resistant bacteria in health care settings: a tale of 2 guidelines. Clin Infect Dis. 2006;42(6):828-835.

20. Vergnano S, Menson E, Kennea N, et al. Neonatal infections in England: the NeonIN surveillance network. Arch Dis Child Fetal Neonatal Ed. 2011;96(1):F9-F14.

21. Stoll BJ, Hansen NI, Sánchez PJ, et al; Eunice Kennedy Shriver National Institute of Child Health and Human Development Neonatal Research Network. Early onset neonatal sepsis: the burden of group B Streptococcal and E. coli disease continues. Pediatrics. 2011;127(5):817-826.

22. Matsubara K, Hoshina K, Suzuki Y. Early-onset and late-onset group B streptococcal disease in Japan: a nationwide surveillance study, 2004-2010. Int J Infect Dis. 2013;17(6):e379-e384.

23. Schrag S, Gorwitz R, Fultz-Butts K, Schuchat A. Prevention of perinatal group B streptococcal disease. Revised guidelines from CDC. $M M W R$ Recomm Rep. 2002;51(RR-11):1-22.

24. Kusuda S, Fujimura M, Sakuma I, et al; Neonatal Research Network, Japan. Morbidity and mortality of infants with very low birth weight in Japan: center variation. Pediatrics. 2006;118(4):e1130-e1138.

25. Isayama T, Lee SK, Mori R, et al; Canadian Neonatal Network; Neonatal Research Network of Japan. Comparison of mortality and morbidity of very low birth weight infants between Canada and Japan. Pediatrics. 2012;130(4):e957-e965.

26. Boghossian NS, Page GP, Bell EF, et al; Eunice Kennedy Shriver National Institute of Child Health and Human Development Neonatal Research Network. Late-onset sepsis in very low birth weight infants from singleton and multiple-gestation births. J Pediatr. 2013;162(6): 1120-1124, 1124. e1.

27. Wu JH, Chen CY, Tsao PN, Hsieh WS, Chou HC. Neonatal sepsis: a 6-year analysis in a neonatal care unit in Taiwan. Pediatr Neonatol. 2009;50(3):88-95.

28. Itabashi K, Horiuchi T, Kusuda S, et al. Mortality rates for extremely low birth weight infants born in Japan in 2005. Pediatrics. 2009;123(2): 445-450.

29. Boyce JM, Pittet D; Healthcare Infection Control Practices Advisory Committee; HICPAC/SHEA/APIC/IDSA Hand Hygiene Task Force. Guideline for Hand Hygiene in Health-Care Settings. Recommendations of the Healthcare Infection Control Practices Advisory Committee and the HICPAC/SHEA/APIC/IDSA Hand Hygiene Task Force. Society for Healthcare Epidemiology of America/Association for Professionals in Infection Control/Infectious Diseases Society of America. MMWR Recomm Rep. 2002;51(RR-16):1-45, quiz CE1-4.

30. Siegel JD, Rhinehart E, Jackson M, Chiarello L; Healthcare Infection Control Practices Advisory Committee. Management of multidrugresistant organisms in health care settings, 2006. Am J Infect Control. 2007;35(10 Suppl 2):S165-S193.

Research and Reports in Neonatology

\section{Publish your work in this journal}

Research and Reports in Neonatology is an international, peer-reviewed, open access journal publishing original research, reports, editorials, reviews and commentaries on neonatal health. The manuscript management system is completely online and includes a very quick and fair

peer-review system. Visit http://www.dovepress.com/testimonials.php to read real quotes from published authors. 